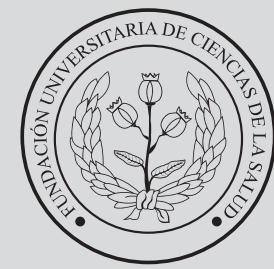

FUCS
Re por

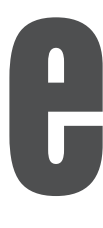

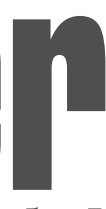
$t$ (1) 10 de Medicina y Cirugía

\title{
Desafios y retos de la fisioterapia desde la visión de las ciencias del movimiento
}

\section{Hurdles and challenges in physiotherapy practice from the movement sciences perspective}

Jorge Enrique Moreno Collazos ${ }^{a}$

${ }^{a}$ Fisioterapeuta. Especialista en rehabilitación cardiopulmonar. Magister en Ciencias de la Actividad Física y Deporte. Doctor en Terapia Manual. Coordinador del Programa de Fisioterapia. Fundación Universitaria de Ciencias de la Salud. Bogotá DC, Colombia.

El movimiento como "sistema" representa en esencia la interacción de los sistemas cardiovascular, pulmonar, endocrino, tegumentario, nervioso y muscular esquelético, que interactúan para mover el cuerpo con sus partes $\mathrm{y} / \mathrm{o}$ componentes. ${ }^{1}$ De allí la importancia de la promoción de la salud a partir de la educación del movimiento como pilar importante en el ciclo de la vida para los procesos de formación de los fisioterapeutas en Colombia, contribuyendo a un cambio en los imaginarios, creencias y prácticas en la emancipación social en lo relativo al objeto de estudio: el movimiento y el cuerpo humano.

La Ley 528 de $1999^{2}$ define a la fisioterapia en Colombia como una profesión liberal del área de la salud, cuyo ejercicio está en manos de profesionales con título universitario; su campo de acción se proyecta a todos los escenarios, ámbitos y lugares en donde el hombre se mueve a lo largo de su vida.

La profesión orienta sus acciones hacia la promoción de la salud, la prevención de la enfermedad, el mantenimiento, optimización o potenciación del movimiento corporal humano en relación con las limitaciones, disfunciones y discapacidades, así como en la participación en procesos interdisciplinarios de adaptación, habilitación y rehabilitación integral de las personas, todo esto con el fin de optimizar su funcionalidad, bienestar, calidad de vida y desarrollo humano social.

El fisioterapeuta es un profesional competente para desempeñarse en las áreas definidas en el perfil profesional, de acuerdo con el talento humano en salud aceptadas universalmente por la comunidad profesional, tales como actividad física y salud, salud y seguridad en el trabajo, salud pública y gestión social, administración y gestión en salud, y educación y atención clínica. ${ }^{3}$ Orienta su práctica profesional con autonomía intelectual y criterio propio en la toma de decisiones, a partir de sólidos fundamentos científicos y férreos principios éticos y humanísticos. Según la Confederación Mundial de Fisioterapia (WCPT por sus siglas en inglés), los fisioterapeutas brindan servicios a individuos y colectivos humanos para conservar o restaurar la máxima capacidad funcional del movimiento a través del ciclo vital humano, como aporte al cuidado integral de la salud. Desde esta perspectiva, la práctica de los fisioterapeutas incluye la provisión de servicios para potencializar el movimiento, la funcionalidad y funcionamiento corporal. ${ }^{4}$

INFORMACIÓN DEL ARTÍCULO

Historia del artículo:

Fecha recibido: agosto 4 de 2019

Fecha aceptado: agosto 14 de 2019
Autor para correspondencia. Jorge Enrique Moreno Collazos jorgemorenoudes@yahoo.com
DOI

10.31260/RepertMedCir.v28.n3.2019.953 
El concepto amplio y contemporáneo de salud al que se acoge hoy la fisioterapia reconoce la importancia de los determinantes sociales de la salud en interacción con las ciencias biológicas, revela la relación entre pobreza, condiciones de trabajo y enfermedad como elementos clave que afectan el proceso de salud y establecen que los procesos sociales macro condicionan los patrones de salud y enfermedad en lo micro. ${ }^{5}$ Por ello, es esencial la participación política de los sujetos en las relaciones de poder para identificar los procesos de transformación social y construir de manera efectiva los de emancipación, desde el movimiento corporal humano como agente de cambio social integrativo y de inclusión. Este papel le compete al profesional de fisioterapia frente a la construcción de respuestas a las nuevas necesidades de salud de la población, en un contexto global que incluya la formación, el desarrollo de la investigación y la transformación de la dinámica de prestación de los servicios.

Es por ello que en la $66^{a}$ Asamblea Mundial de la Salud de 2013, ${ }^{6}$ se destacó la necesidad de comprender los vínculos entre la salud y el desarrollo sostenible. En este marco han ganado importancia las cuestiones relativas con la equidad, rescatando la necesidad de retomar la estrategia de la cobertura sanitaria universal para reducir las inequidades. Dentro de los retos mundiales se destacan los vinculados con el proceso de cambio climático, la garantía de agua y de ambientes saludables, alimentación adecuada y financiamiento necesario para el sector, entre muchos otros. ${ }^{7}$ Así mismo, entre los retos sanitarios se destacan el aumento de las enfermedades no transmisibles, la necesidad de la atención a la salud mental, la preocupación por el logro de una vejez saludable y el control de los eventos imprevistos que afectan la salud en el mundo.

La Fundación Universitaria de Ciencias de la Salud en respuesta a los desafíos de la salud en Colombia presenta su nuevo programa de fisioterapia, enfocado a la capacitación de talento humano en el ámbito del movimiento corporal humano en las áreas músculo esquelética, neuromuscular e integumentaria, con énfasis en el concepto de la fisioterapia cardiovascular-pulmonar. Está articulado con las tendencias de la actividad física en salud y el bienestar cinético en las poblaciones vulnerables, así como en los estadios agudos y crónicos cardiovasculares y pulmonares, promoviendo la formación de fisioterapeutas con valores y calidad académica y científica, iniciando en el pregrado hasta llegar a los estudios de posgrado con tecnología y enfoque vanguardista de la práctica, basada en la evidencia científica para lograr el bienestar cinético en el ciclo de la vida.

\section{REFEREN CI AS}

1. Voight ML, Hoogenboom BJ. What Is the Movement System and Why Is It Important?. Int J Sports Phys Ther. 2017;12(1):1-2.

2. Ley 528, por la cual se reglamenta el ejercicio de la profesión de fisioterapia, se dictan normas en materia de ética profesional y otras disposiciones. , Diario Oficial 43.711 (1999).

3. Asociación Colombiana de Fisioterapia (ASCOFI), Asociación Colombiana de Facultades de Fisioterapia (ASCOFAFI), Colegio Colombiano de Fisioterapeutas (COLFI), Asociación Colombiana de Estudiantes de Fisioterapia (ACEFIT). Perfil profesional y competencias del fisioterapeuta en Colombia. Bogotá: Ministerio de Salud y Protección Social; 2015. p. 62.

4. Ramírez-Vélez R, Escobar Hurtado C, Florez López ME. Análisis de la capacidad científica e investigativa de los profesionales en Fisioterapia de Colombia. Dificultades y oportunidades de desarrollo. Rev Iberoam Fisioter Kinesio. 2010;13(1):37-45.

5. World Confederation for Physical Therapy. WCPT guideline for physical therapist professional entry level education [Internet]. London: World Confederation for Physical Therapy; 2007 [citado 2019 agosto]; Recuperado de: https://www.wcpt.org/guidelines/ entry-level-education.

6. Slade SC, Hay-Smith J, Mastwyk S, Morris ME, Frawley H. Attributes of physiotherapy continence clinicians: a qualitative perspective. Physiotherapy. 2019.

7. Organización Mundial de la Salud. La 66. ${ }^{a}$ Asamblea Mundial de la Salud, resoluciones y decisiones. Ginebra: Organización Mundial de la Salud; 2013. p. 210. 